SUPPORTING INFORMATION

\title{
Effect of methylation level on grape fruit development process
}

Haoran Jia, Zhibo Zhang, Saihang Zhang, Weihong Fu, Haifeng Jia*, Jinggui Fang

Author Affiliations

College of Horticulture, Nanjing Agricultural University, Nanjing 210095, China

*Corresponding author: Haifeng Jia

Email: jiahaifeng@njau.edu.cn 

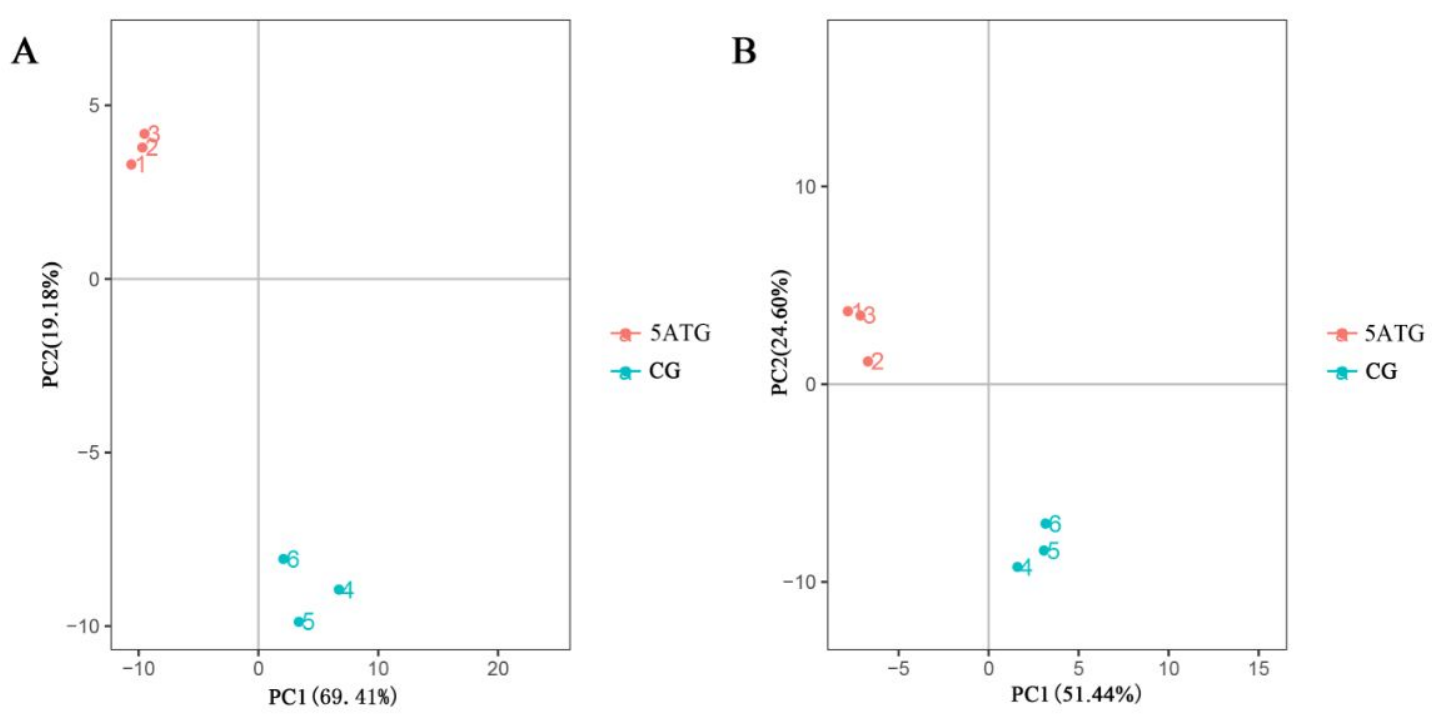

Fig S1.

PCA analyzed the difference in metabolome between 5MTG and CG in positive (A) and negative (B) ion modes. 

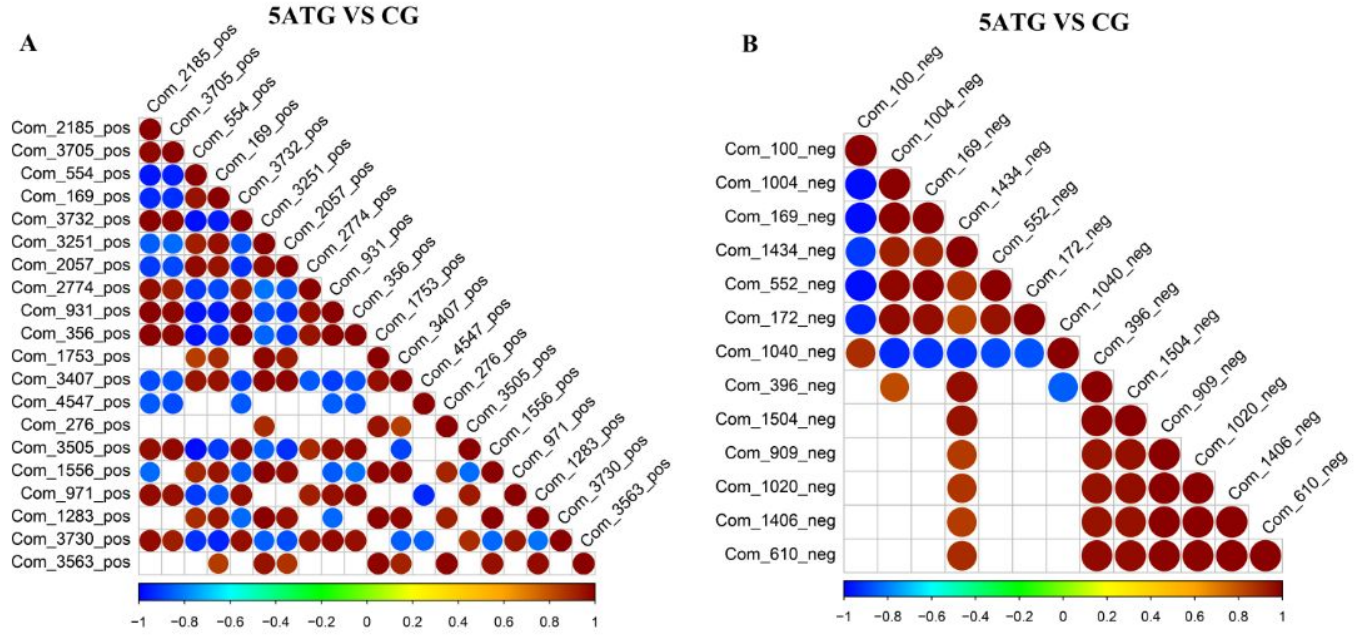

Fig S2

Correlation analysis of differentially expressed metabolites. A. Positive, B. Negative. 
A

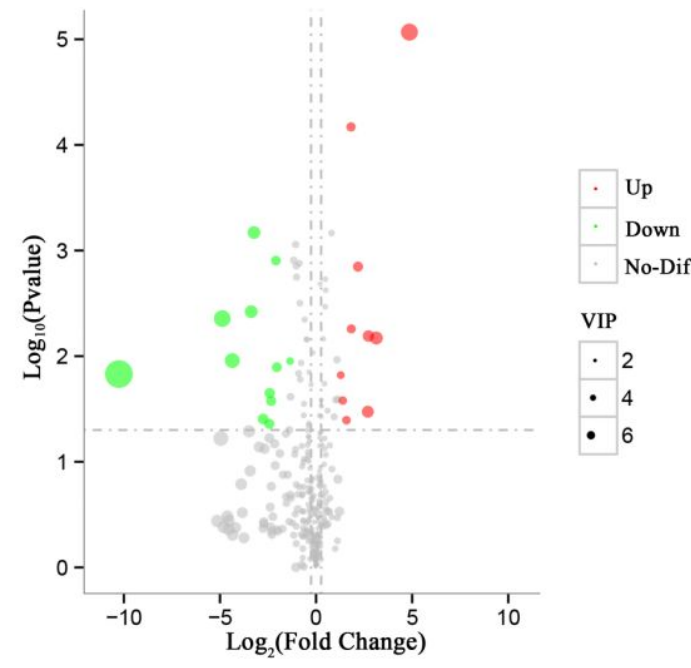

B

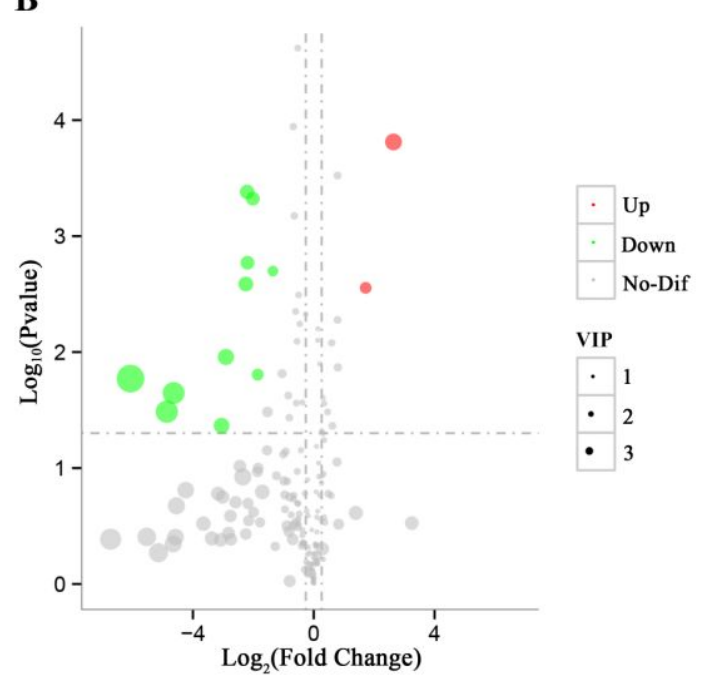

Fig S3.

Volcano plot analyzing differential expression of metabolites in 5MTG and CG. A. Positive, B. Negative. 


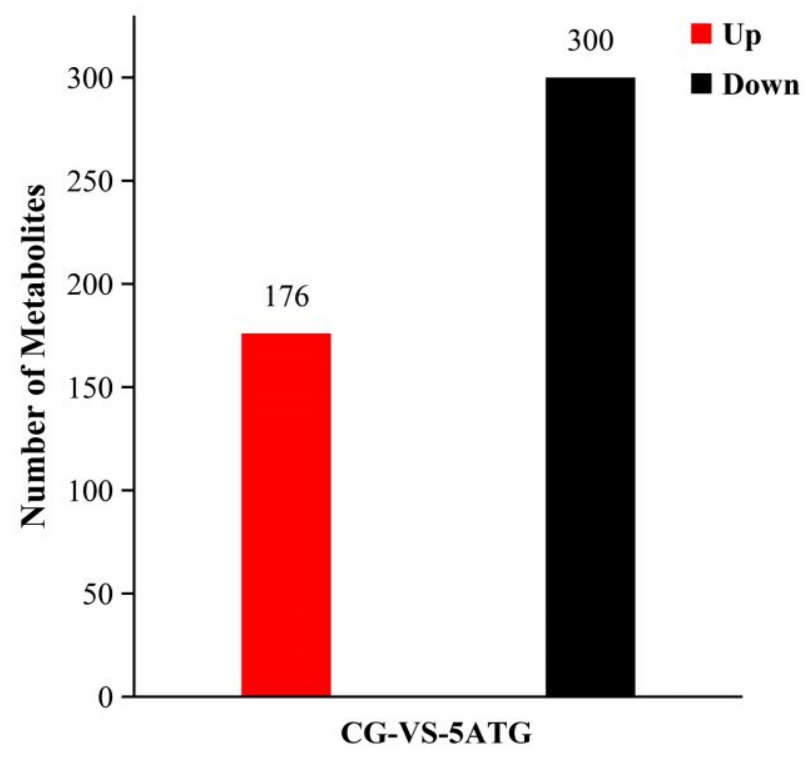

Fig S4.

Statistics of differential metabolites. 


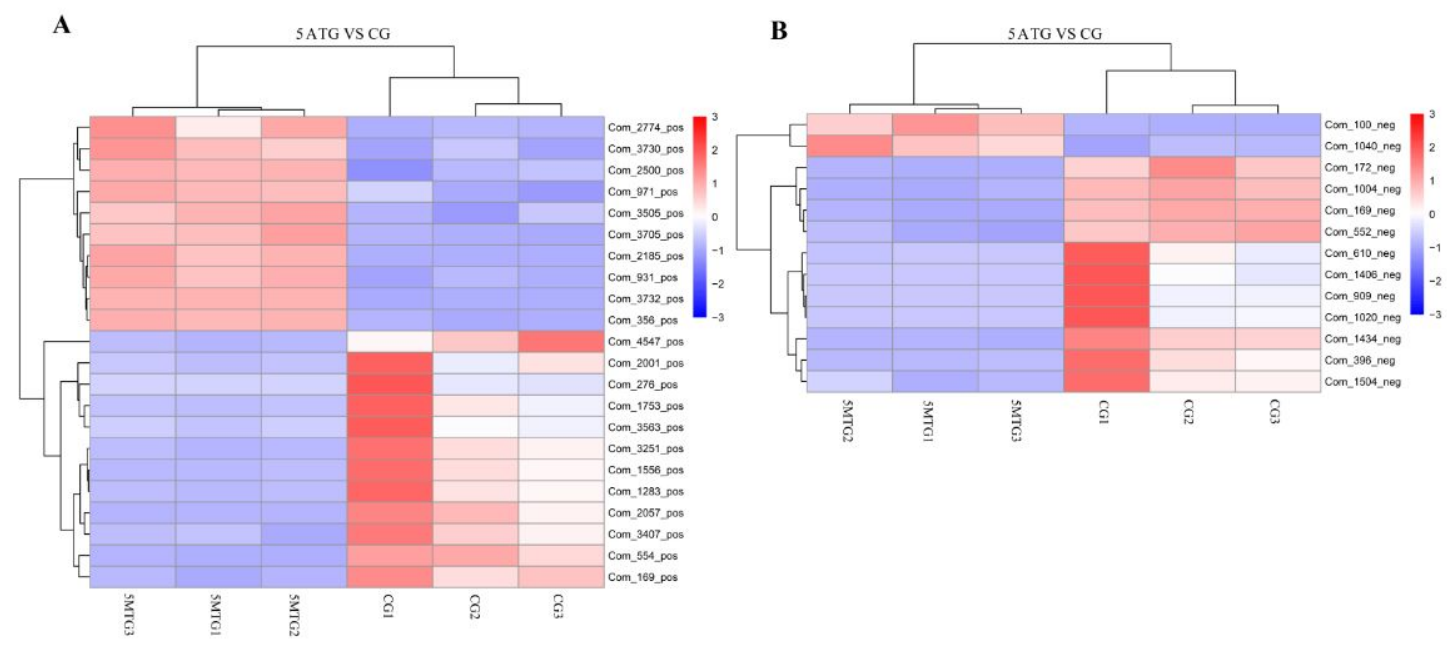

Fig S5.

Differential metabolites cluster heat map. A. Positive, B. Negative. 
A

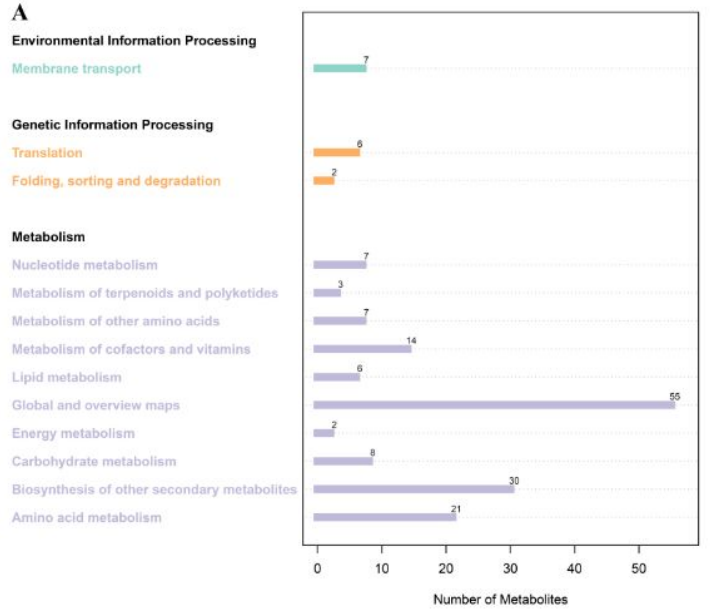

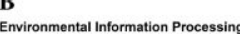

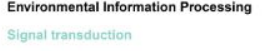

Membrane transpo

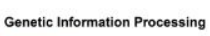

Metabolism

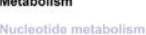

Wetaholism of terpenoids and polyketictes

Metabolism of other amino acids

Metabolism of cofactors and vitamin:

Lipid metabolism

overviow maps

Chergy metabolism

Cemoriyarate metabolism

Biosynthesis of other sectonda

Fig S6.

KEGG function annotation of differential metabolites. A. Positive, B. Negative. 

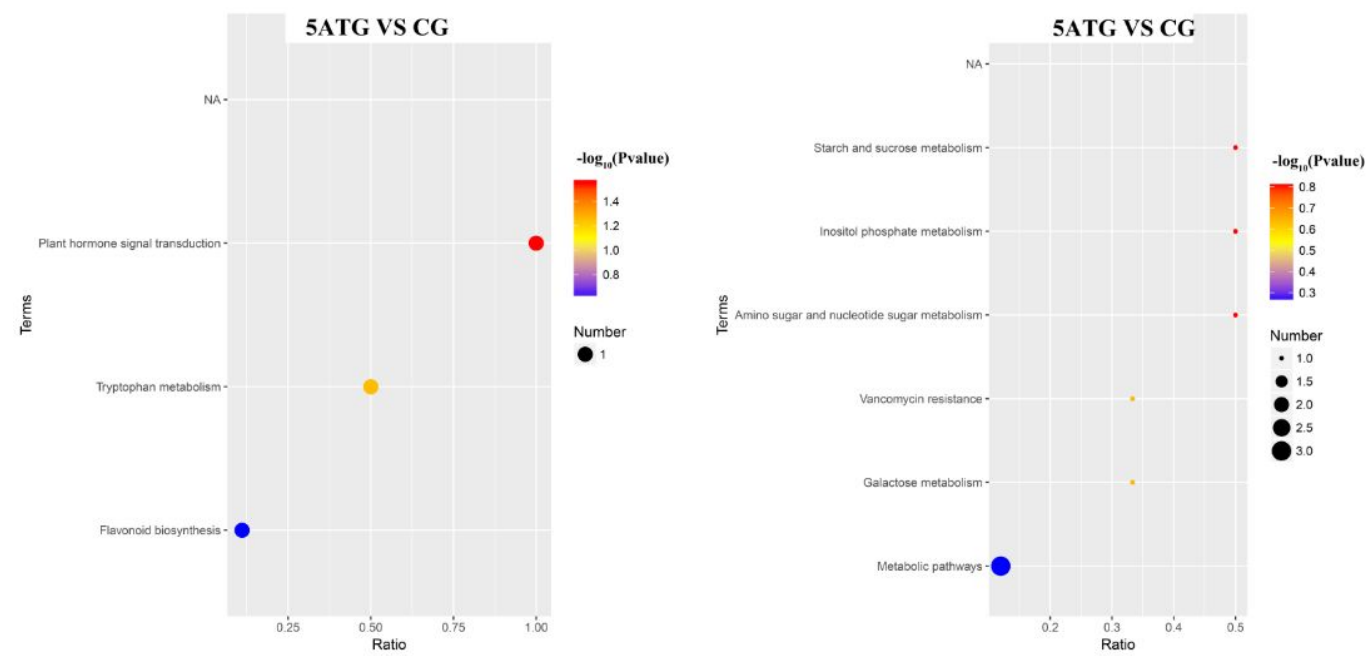

Fig S7.

KEGG enriched bubble chart of differential metabolites. A. Positive, B. Negative. 

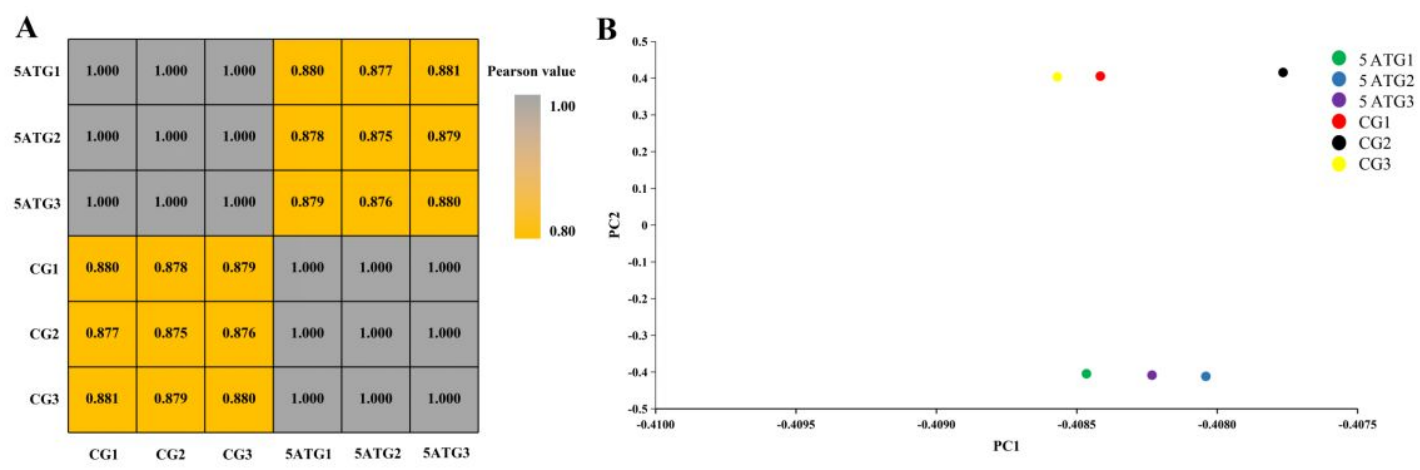

\section{Fig S8.}

Correlation matrix (A) and PCA (B) analysis of DEGs were performed on three biological replicates of each sample set (5MTG and CG) to evaluate correlations between samples. 


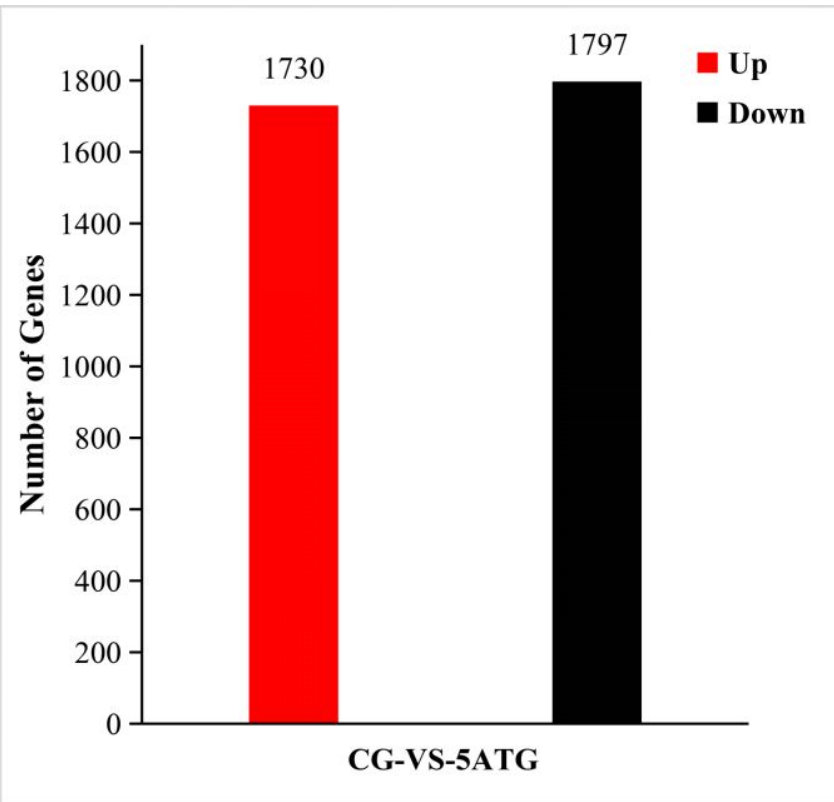

Fig S9.

Differential genes quantity statistics. Statistics were performed on up-regulated and down-regulated genes between CG-vs-5MTG. 


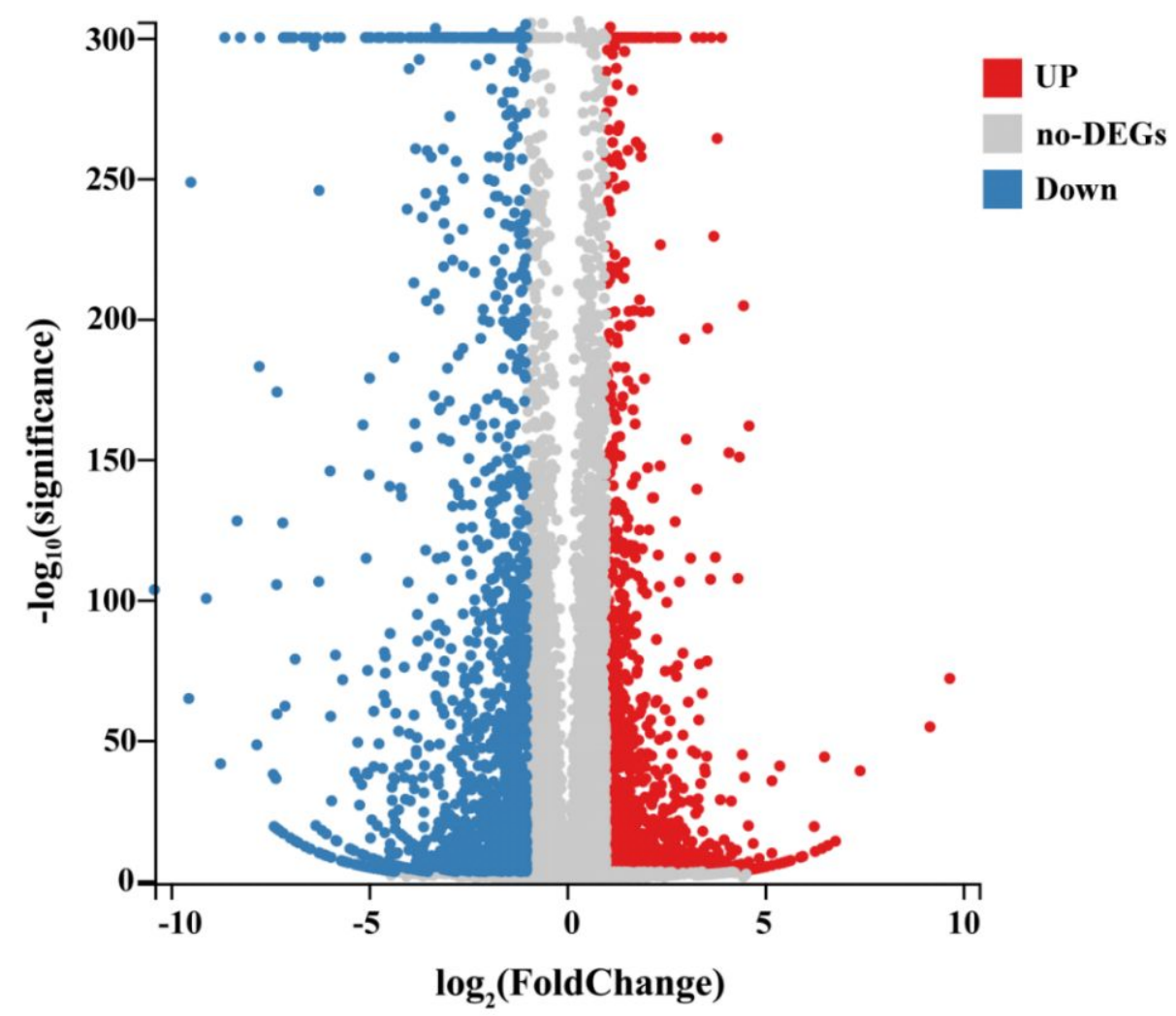

Fig S10.

The expression profiles of the identified DEGs. The $\mathrm{X}$ axis represents the difference multiple of the $\log _{2}$ (conversion), the $\mathrm{Y}$ axis represents the significance value after the $-\log _{10}$ (conversion). The up and down DEGs are represented by red and blue dots, respectively, gray represents non-DEGs. 